\title{
Association Between Appropriate Complementary Feeding and Anthropometric Measures Among Infants and Young Children in Malawi using Cross- Sectional Data: A Propensity Score Matching Approach
}

Halima Sumayya Twabi ( $\nabla$ htwabi@cc.ac.mw)

University of Malawi Chancellor College https://orcid.org/0000-0002-0166-9393

Samuel $O$ Manda

South Africa Medical Research Council

Dylan Small

University of Pennsylvania

Research article

Keywords: Propensity score, Complementary feeding, Survey, Children

Posted Date: September 21st, 2020

DOl: https://doi.org/10.21203/rs.3.rs-53425/v1

License: (1) (i) This work is licensed under a Creative Commons Attribution 4.0 International License.

Read Full License 


\section{RESEARCH}

Association between appropriate complementary feeding and anthropometric measures among infants and young children in Malawi using cross-sectional data: A propensity score matching approach

Halima S Twabi ${ }^{1 *}$, Samuel O Manda ${ }^{2,3,4}$ and Dylan S Small ${ }^{5}$ 
Correspondence:

twabi@cc.ac.mw,

muel.manda@mrc.ac.za

small@wharton.upenn.edu Department of Mathematical ciences, University of Malawi, omba, Malawi

ull list of author information is vailable at the end of the article Equal contributor

\begin{abstract}
Background: Inappropriate feeding practices among infants and young children are adversely associated with their growth and development. This study aimed to investigate the causal association between appropriate complementary feeding and child growth among children aged 6 to 23 months in Malawi using propensity score matching.

Methods: Data on 4722 children aged 6 to 23 months from the 2015-16 Malawi Demographic and Health Survey (MDHS) were analysed. Distribution of confounder variables on children who were not on appropriate complementary feeding were made similar to those who were on appropriate complementary feeding using a 1:1 nearest neighbour matching within a calliper region of 0.1 . Matching was evaluated based on the average standardised absolute mean differences and covariate specific p-values after matching. The effect of appropriate complementary feeding was estimated based on the matched sample. A sensitivity analysis was done to assess the effect of unmeasured confounders on the estimates.
\end{abstract}

Results: The prevalence of stunting was at $28 \%$ (95\% Cl:27\%,29\%), wasting was at $3.3 \%(95 \% \mathrm{Cl}: 2.5 \%, 4.1 \%)$ and underweight was at $13.6 \%(95 \% \mathrm{Cl}: 12.1 \%, 15 \%)$. Of the 4772 children, $16.7 \%(95 \% \mathrm{Cl}: 15.6,17.8)$ were given appropriate complementary feeding. A majority $(81.8 \%)$ of the children were initiated to complementary feeding after 6 months of age. Appropriate complementary feeding was associated with reduced stunting ( $\mathrm{OR}=0.5,95 \% \mathrm{Cl}: 0.3,0.9)$ among the children but did not have an effect on wasting and underweight.

Conclusion: The study has found that appropriate complementary feeding led to less stunting among children aged 6-23 months in Malawi. The use of statistical techniques such as propensity score matching to balance confounder variables could be useful to reduce bias in the estimation of exposure comparative effectiveness from observational studies. We recommend optimal feeding for young children, especially in resource-limited settings.

Keywords: Propensity score; Complementary feeding; Survey; Children

\section{Background}

Child under-nutrition is one of the leading causes of under-five mortality in the world. According to the World Health Organisation (WHO) [1], 2.7 million child 
deaths worldwide, which makes up $45 \%$ of all infant mortality are associated with under-nutrition. The burden of child under-nutrition is high in developing countries, particularly among those in Sub-Saharan Africa (SSA) [2]. In 2016, more than one-third of stunted children (38\%) and more than one-quarter of wasted (27\%) children lived in sub-Saharan Africa [3]. Anthropometric measurements such as weight, height (or length), sex, head-circumference, mid upper arm circumference (MUAC) and age are recorded from a child to determine and evaluate their growth and nutritional status. These measurements grouped together form anthropometric indices [4] such as head-circumference-for-age and body mass index-for-age. In this study we focus on the three most commonly used indices namely; height-for-age, weight-for-age and weight-for-height [5].

The WHO recommends exclusive breastfeeding and appropriate complementary feeding as the optimal infant feeding practice for children under two years of age to ensure good child growth and development [6-8]. Complementary feeding entails giving a child other semi-solid, soft-foods and solid foods, liquids, water along with breast milk from the age of 6 to 23 months [9]. Appropriate complementary feeding involves initiating complementary feeding at the appropriate time, providing the appropriate food groups and feeding a child sufficiently [9]. The Malawi government (MG) implemented guiding principles on infant and young child feeding since the early 2000s [10]. The overall objective of the policy and guidelines is to improve infant and young child nutrition for survival, growth and development [10]. Adherence to the guidelines by mothers or care takers tends to be a challenge due to factors such as poverty, poor water, sanitation and hygiene (WASH) practices and cultural norms $[11,12]$. Coupled to lack of resources to practice appropriate child feeding, prior evidence has shown that some cultures leave child feeding decisions to mothers while in other cultures, the immediate relatives influence this decision [12].

Malawi is one of the countries in SSA that has a high burden of HIV/AIDS which has contributed to adverse effects on child health and growth [13]. In Malawi, the Prevention of Mother to Child HIV transmission (PMTCT) was integrated in the guidelines on infant and young child feeding [10] to ensure optimal growth among children born to HIV-infected mothers. There is disparity on the evidence of com- 
plementary feeding on child growth in low-and middle-income countries (LMICs). Some studies have found no association between dietary diversity and any anthropometric failure [14]. Other studies found that complementary feeding led to an increase in underweight and and a decrease in stunting, while others have shown that adequate dietary diverse foods were negatively associated with stunting $[15,16]$. Furthermore, previous studies have found an association between infant and young child feeding and child growth in the context of HIV-infection in resource limited countries using observational data [17-19].

Population and household-based surveys provide most of the child health indicators in low and middle income countries [20]. However, observational data render an assessment of causal inference limited as there could be other purported factors such as maternal education, duration of breastfeeding, source of drinking water, household wealth, child's age maternal body mass index, employment and media exposure [21-24], besides feeding practices and maternal HIV, associated with child growth. The presence of these factors misrepresent the impact of appropriate complementary feeding on infant and young child growth as the distribution of these factors may be different between the exposure groups. To assess causal association, a randomised control trial (RCT) would ideally need to be conducted. However, since most data on child health outcomes are observational, one could still achieve the same task of balancing confounder variables between the exposure variable apprpriate complementary feeding by using methods developed by Rosenbaum et al.,[25]. Propensity Score (PS) methods were developed with the aim of emulating the randomisation procedure of RCTs, as the distribution of measured confounders between exposure groups is made similar by using the PS [25-35].

Despite the efforts to implement the policies and guiding principles on infant and young child feeding, the prevalence of under-nutrition remains high in Malawi as compared to other Sub-Saharan African (SSA) countries [2, 36]. Although previous studies have assessed child complementary feeding and child growth in Malawi, including in an HIV context [11, 12,37], these studies used observational data which are prone to confounder bias and balancing methods such as the propensity score methods were rarely used. Therefore, the study aimed to assess the causal association between appropriate complementary feeding and child growth using 
statistical tools that control for confounding. This study provides an understanding of how purported infant and young child feeding impacts child growth, which in turn provides reliable evidence to policy-makers.

\section{Methods}

\section{Study Data and Design}

The data was obtained from the 2015-16 Malawian Demographic Health Survey (MDHS), which was implemented by the National Statistical Office of Malawi in conjunction with the Ministry of Health and the Community Health Services Unit of Malawi. As with other demographic health surveys (DHS), the 2015-16 MDHS used a multi-stage stratified sampling design where first stage sampling involved a random selection of enumeration areas (clusters) as primary sampling units and households in the second stage sampling. Women aged 15-49 years and men aged 15-54 years were eligible to participate in the survey. At the first stage, the cluster sampling frame was stratified by geographic type (rural/urban) and districts. A total of 850 clusters (173 and 677 from urban and rural areas, respectively) were selected at the first stage with selection probability proportional to the cluster size and independent at each sampling stratum. In all, 26,361 households were selected using random systematic sampling. More details on the sampling procedure and design can be obtained from the 2015-16 MDHS report [36].

The study analysed data on 4722 children aged 6 to 23 months. A child's weight (in kilograms) and height (in centimetres) were measured in one-third of sampled households. Weight was measured with an electronic scale and very young children were weighed by weighing the mother first and then the mother was weighed again while holding the child. Using an automatic two-in-one adjustment button allowed the mother's stored weight to be deducted and the infant's weight was recorded. Height was measured with a Shorr Board $₫$ measuring board [36]. Recumbent length was measured for children less than 24 months of age and for older children standing height was measured. Children's height or length, weight, and age data were used to calculate three anthropometric indices: height-for-age, weight for-height, and weight-for-age. 


\section{Data collection for maternal HIV}

Collection of HIV data from the survey was obtained from the participants by collecting finger-prick blood specimens from women aged 15-49 years and men aged 15-54 years who consented to laboratory HIV testing by the interviewers. The testing procedure, confidentiality of the data, and the fact that the test results would not be made available to respondents was explained by the interviewer to the participants. Further details on data collection for HIV data can be found in the 2015-16 MDHS report [36]. Mothers HIV status in this study was recoded as $(0=$ HIV negative, $1=$ HIV positive).

\section{Outcome and Confounder Variables}

The three common anthropometric indices of measuring a child's nutritional status height-for-age z-score (HAZ), weight-for-height z-score (WHZ) and weight-for-age z-score (WAZ) were used as outcome variables in this study. These indices were derived as a z-score by comparing a child's height/length or weight and age with the median value in the reference population. The difference is then divided by the standard deviation of the reference population [38]. The 2015-16 MDHS used the 2006 WHO median values for height and weight at different age groups as reference values [38]. Normal nutritional status is defined as having a measurement of -2.0 or greater. If the calculated z-score of a child falls below the -2 cut-off point, then the child is 2 standard deviations below the average and is considered to be undernourished [38].

We defined stunting=1 if HAZ was below minus two standard deviation (-2SD) from the median of the reference population and 0 otherwise $(1=\mathrm{HAZ}<-2 \mathrm{SD}$ and $0=\mathrm{HAZ} \geq-2 \mathrm{SD}$ ). Wasting $=1$ if $\mathrm{WHZ}$ was below minus two standard deviation (-2SD) from the median of the reference population and 0 otherwise $(1=\mathrm{WHZ}<-2$ $\mathrm{SD}$ and $0=\geq-2 \mathrm{SD}$ ), and underweight $=1$ if WAZ was below minus two stan-

dard deviation (-2SD) from the median of the reference population and 0 otherwise $(1=\mathrm{WAZ}<-2 \mathrm{SD}$ and $0=\mathrm{WAZ} \geq-2 \mathrm{SD})$.

Several variables were identified from the MDHS as potential confounders on complementary feeding and child growth. These include maternal age, wealth index, place of residence, history of diarrhoea, birth weight, breastfeeding status of a child, maternal education, sex of a child and age of a child. In addition, mothers 


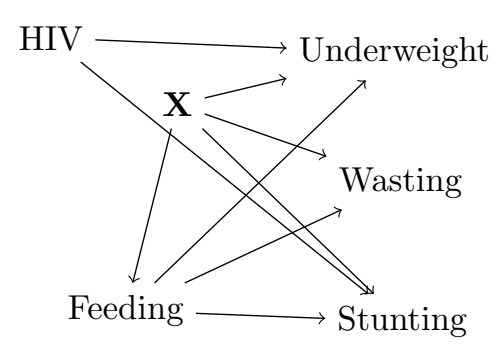

Figure 1 Causal pathway for Appropriate Feeding and Child Nutrition

employment, initiation of complementary, types of foods, counselling of complementary feeding, birth weight, duration of breastfeeding, antenatal care and having diarrhoea were also identified as potential confounders. However, we replaced mothers' employment status with mothers' education and wealth as only a few mothers were full-time employed, hence the matched sample would have been small within the full time category.

Figure 1 shows the causal pathway of appropriate complementary feeding effect on child growth. Maternal HIV-infection has been shown to affect a child's growth $[18,39]$, hence we postulate a path from HIV to wasting, stunting and underweight. Since child growth may differ in children due to maternal HIV but the decision to practice appropriate complementary feeding was not influenced by maternal HIV since mothers in the 2015-16 MDHS did not know their HIV test result. Therefore, we balanced the differences between children who were appropriately complementary fed and those who were not appropriately complementary fed by maternal HIV-infection. The causal pathway in Figure 1 therefore shows the paths of the causal association and the confounder effect on appropriate complementary feeding.

\section{Measurement of complementary feeding indicators}

The World Health Organisation (WHO) through the Global Strategy for Infant and Young Child Feeding [7] defines complementary feeding as giving a child semi-solid and solid foods, liquids, water along with breast milk from the age of 6 to 24 months. Complementary feeding was defined using the core key indicators recommended by the WHO/UNICEF in 2008 [8] which involves introduction of complementary feeding, minimum dietary diversity, minimum meal frequency and minimum acceptable 
diet based on a dietary intake 24 hours before the survey and calculated for the age ranges 6-11, 12-17 and 18-23 months of age. These are defined as:

i) Timely introduction of complementary feeding $=1$, if a child aged 6-23 months started complementary foods (solid, semi-solid or soft) at 6-8 month of age and 0 otherwise $[8,21]$.

ii) Minimum dietary diversity (MDD) $=1$ if a child received foods from four or more food groups during the previous day and 0 otherwise. This refers to the child receiving the following food groups; grains, roots and tubers; legumes and nuts; dairy products (milk, yoghurt and cheese); flesh foods (meat, fish, poultry and liver/organ meats); eggs; vitamin A-rich fruits and vegetables; and other fruits and vegetables $[8,21]$.

iii) Minimum meal frequency $(\mathrm{MMF})=1$ if a breastfeeding and non-breastfeeding child aged 6-23 months received complementary foods the minimum number of times or more (minimum is defined as: two times for breastfed infants 6-8 months; three times for breastfed children 9-23 months; and four times for non-breastfed children 6-23 months) in the previous day [8, 21], 0 otherwise.

iv) Minimum acceptable diet $(\mathrm{MAD})=1$ if a child was fed a minimum dietary diversity and minimum meal frequency during the day or night preceding the survey $[8,36]$ and 0 otherwise.

We adopted Kassa et al's., [21] quantification of appropriate complementary feeding practise by considering core WHO infant and young child feeding indicators from the MDHS data. These are timely introduction of complementary feeding, minimum dietary diversity and minimum meal frequency. If a child had timely introduction of complementary feeding and minimum dietary diversity and minimum meal frequency, the child was classified as having appropriate complementary feeding, (appropriate feeding $=1$ ) otherwise (Not appropriate feeding $=0$ ).

\section{Handling missing data}

A descriptive analysis on the data indicated that the MDHS data had more than $10 \%$ of missing data. Therefore, we conducted a multiple imputation on the data, using the mice package in R. All statistical analyses were done on the imputed data [40]. 


\section{Statistical Analysis}

We used the propensity score matching (PSM) to compare children on appropriate complementary feeding and those not on appropriate complementary feeding (CF) groups, in effect, mimicking a randomised control trial (RCT). Children who were appropriately $\mathrm{CF}$ were matched to those who were not appropriately $\mathrm{CF}$ with similar characteristics using a propensity score (PS). The propensity score given observable characters $X$ is denoted as [25];

$$
\pi_{i}\left(x_{i}\right)=\operatorname{Pr}\left(A_{i}=1 \mid X_{i}\right)
$$

Where $A_{i}=1$ if a child is appropriately CF. The PS, estimated using a logit or a probit model, estimates the likelihood of a child being in the appropriate complementary feeding group. Matching the children using a PS is equivalent to matching on each observable characteristics [29].

Rosenbaum et al. [25] proved that the propensity score is a balancing score and conditioning on the PS, the potential outcomes are independent of exposure. Variables that were identified as potential confounders were fit in the logistic regression model to estimate the propensity scores. A forward model selection was applied to select potential interactions. The Hosmer and Lemeshow goodness-of-fit test was performed to check if the model fits the data well. We used a nearest neighbour algorithm with a 0.1 calliper to restrict the difference in PS between matched children.

Some studies [41], have shown that matching and inverse probability weighting eliminated systematic differences between exposure groups to a greater degree than stratification or covariate adjustment and that their estimates were close to those of randomised control trials (RCTs). Hence, this study used the PS matching to control for confounding. The association between appropriate complementary feeding and each of the three child growth indicators was measured by an odds ratio (OR). The strongest assumption of the PS matching is that there remains no unobserved confounding. It is impossible to prove that no unobserved confounding exists but through a sensitivity analysis [42] we measure if the results are sensitive to hidden bias. 


\section{Covariate Balance}

The standardised prevalence difference was used to assess balance for the distribution of measured potentially confounded covariates between appropriately and inappropriately complementary fed children. We compared the prevalence [43] of the categorical covariates in appropriately $\mathrm{CF}$ and inappropriately $\mathrm{CF}$ children across the measured covariates. A standardised difference of 0.1 (10 per cent) was used as a decision criterion and denoted meaningful balance in the measured covariates $[40,44]$. We further check for balance by ensuring that each confounder does not significantly differ in proportion between children who were appropriately complementary fed and those who were not appropriately complementary fed using a chi-square test for categorical variables.

\section{Sensitivity Analysis}

It is impossible to prove that no unobserved confounding exists, [42] hence we perform a sensitivity analysis to assess the robustness of the results. The MantelHaenszel (MH) non-parametric test statistics was used to assess the sensitivity of the estimates to unmeasured confounders. The $\mathrm{MH}$ non-parametric test compares the successful number of children in the appropriate $\mathrm{CF}$ group with the same expected number, given that the appropriate $\mathrm{CF}$ effect is zero [45]. The maximum value of $\Gamma$, a range of possible values attributable to unobserved heterogeneity, was set from 1 to 3 with an increment of 0.2 . A value of gamma close to 1 and significant indicates sensitivity to unobserved heterogeneity [46]. Sensitivity analyses were conducted for all outcome variables. We used the mhbounds package to obtain the values of the upper and lower bounds on the estimates. 


\section{Results}

Summary information about feeding practices of the 4277 studied children aged 6-23 months is presented in Table 1. Of the 4722 children, 4184 (88.5\%) were still breastfeeding while $543(11.5 \%)$ were not breastfeeding at the time of the survey. A majority of the children $3867(81.8)$ were on complementary feeding and 2559 $(66.2 \%)$ of the children started complementary feeding at the age of 12-23 months. A majority $(57.5 \%)$ of the children were fed more than two times a day, the day preceding the survey. The common dietary food given to the children was grains (24.1\%) followed by legumes and nuts (21.5\%). Out of the 4722 children, 3942 (83.6) children had mothers who were counselled on breastfeeding. Combining the three feeding indicators presented in Table 2, the overall prevalence of appropriate complementary feeding practice was $16.7 \%$ (95\% CI:(15.6, 17.8)). For the 2015-16 MDHS data, the overall prevalence of wasting in children under 5 years was $3.3 \%$ (95\% CI: $2.5 \%, 4.1 \%)$, stunting was $28 \%(27 \%, 29 \%)$ and underweight was $13.6 \%$ (95\% CI: $12.1 \%, 15 \%)$.

Indicators of complementary feeding were also assessed as presented in Table 2 . Of the 4722 children, 1349 (28.5\%) children aged 6 to 23 months received solid, semi-solid or soft foods the minimum number of times meeting a minimum meal frequency, 1046 (22.1\%) children were offered four or more food groups criteria on the day preceding the survey meeting the minimum dietary diet and 340 (18.2\%) children were given the minimum adequate diet. The HIV prevalence among women aged 15-49 years was estimated as $9.2 \%$ (95\% CI: 8.4,10.1) in 2015-16.

\section{Distribution of confounders and balance assessment}

Table 3 presents distribution of confounders before and after matching. Out of the $4722(82.8 \%)$ children, 788 (16.7\%) were appropriately complementary fed and 3934 (83.3\%) were not appropriately complementary fed. A fifth $(21.3 \%)$ of the children born to HIV-infected mothers were appropriately complementary fed, while $18.9 \%$ were not appropriately complementary fed. Of the 4722 children, 368 (45.6\%) of the male children were appropriately complementary fed while 2047 (52.0\%) were not appropriately complementary fed. Six-hundred and seventy two $(83.2 \%)$ and $3487(88.6 \%)$ children living in the rural area were appropriately complementary fed and inapprorpiately complementary fed, respectively. 
Table 1 Feeding practices of children from the 2015-16 MDHS

\begin{tabular}{|c|c|c|c|}
\hline \multirow[b]{2}{*}{ Characteristic } & \multicolumn{2}{|c|}{ MDHS 2015-16 } & \multirow[b]{2}{*}{$(95 \% \mathrm{Cl})$} \\
\hline & Freq. & Percent & \\
\hline \multicolumn{4}{|l|}{ Ever breastfed } \\
\hline Yes & 4679 & 98.98 & $(98.7,99.3)$ \\
\hline No & 48 & 1.02 & $(0.7,1.3)$ \\
\hline \multicolumn{4}{|l|}{ Still Breastfeeding } \\
\hline Yes & 4184 & 88.5 & $(87.6,89.4)$ \\
\hline No & 543 & 11.5 & $(10.6,12.4)$ \\
\hline \multicolumn{4}{|c|}{ Current age of child still breastfeeding (in 2015-16) } \\
\hline 6-11 months & 1514 & 36.2 & $(34.7,37.7)$ \\
\hline $12-17$ months & 1485 & 35.5 & $(34.0,36.9)$ \\
\hline $18-23$ months & 1185 & 28.3 & $(26.9,29.7)$ \\
\hline \multicolumn{4}{|c|}{ Current age of child not being breastfed (in 2015-16) } \\
\hline 6-11 months & 103 & 19.0 & $(15.7,22.3)$ \\
\hline $12-17$ months & 152 & 27.9 & $(24.1,31.7)$ \\
\hline $18-23$ months & 288 & 53.1 & $(48.9,57.3)$ \\
\hline \multicolumn{4}{|l|}{ Counselled on BF } \\
\hline Yes & 3942 & 83.6 & $(82.5,84.6)$ \\
\hline No & 762 & 16.2 & $(15.1,17.2)$ \\
\hline Don't know & 14 & 0.3 & $(0.1,0.5)$ \\
\hline Missing & 10 & $(0.2)$ & $(0.09,0.4)$ \\
\hline \multicolumn{4}{|c|}{ Ever started complementary } \\
\hline Yes & 3867 & 81.8 & $(80.7,82.9)$ \\
\hline No & 860 & 18.2 & $(17.1,19.3)$ \\
\hline \multicolumn{4}{|c|}{ Current age of child on complementary feeding (in 2015-16) } \\
\hline 6-11 months & 1301 & 33.8 & $(32.3,35.3)$ \\
\hline $12-17$ months & 1412 & 36.7 & $(35.2,38.2)$ \\
\hline $18-23$ months & 1134 & 29.5 & $(28.0,30.9)$ \\
\hline \multicolumn{4}{|c|}{ No. of times a child aged 6-23 months fed } \\
\hline Once only & 1440 & 30.5 & $(29.2,31.8)$ \\
\hline 2-3 times & 2715 & 57.5 & $(56.1,58.9)$ \\
\hline $4+$ times & 567 & 12 & $(11.1,12.9)$ \\
\hline \multicolumn{4}{|l|}{ Type of dietary food } \\
\hline Diary & 820 & 17.1 & $(15.9,18.1)$ \\
\hline Grain & 1141 & 24.1 & $(22.8,25.3)$ \\
\hline Legumes and nuts & 1021 & 21.5 & $(20.4,22.7)$ \\
\hline Meat & 585 & 12.3 & $(11.4,13.3)$ \\
\hline Eggs & 255 & 5.4 & $(4.7,6.0)$ \\
\hline VA rich fruits and vegs & 89 & 1.9 & $(1.5,2.3)$ \\
\hline Other rich fruits and veg & 21 & 0.4 & $(0.3,0.6)$ \\
\hline
\end{tabular}

BF - Breastfeeding

VA - Vitamin A

Table 3 shows the distribution of the available purported confounder variables of child growth before and after matching. Maternal HIV infection, sex of a child, household wealth index, mothers' educational status and mothers' age were dis- 
Table 2 Proportion of infants and young children meeting four WHO/UNICEF feeding indicators in 2015-16

\begin{tabular}{lcc}
\hline & \multicolumn{2}{c}{ 2015-16 MDHS } \\
\cline { 2 - 3 } Indicator & Freq. & Percent(95\% CI) \\
\hline Minimum adequate diet & & \\
Yes & 340 & $7.2(6.5,7.9)$ \\
No & 4382 & $92.8(92.1,93.5)$ \\
Minimum meal frequency & & \\
Yes & 1349 & $28.5(27.2,29.8)$ \\
No & 3378 & $71.5(70.2,72.8)$ \\
Minimum dietary diversity & & \\
Yes & 1045 & $22.1(16.0,17.9)$ \\
No & 3682 & $77.9(76.7,79.1)$ \\
Time introduction of complementary foods & & \\
Yes & 821 & $21.6(20.2,22.8)$ \\
No & 3618 & $78.5(77.2,79.8)$ \\
\hline
\end{tabular}

tributed differently between appropriately and inappropriately complementary fed children $(P<0.05)$. After PS matching on the confounders, 788 matched pairs (appropriate and inappropriate $\mathrm{CF}$ pair of children) were analysed for difference in baseline characteristics. We observe no significant difference in the distribution of the covariates between appropriately and inappropriately complementary fed children $(P>0.05)$. Furthermore, Table 5 in the appendix presents the absolute standardised differences before and after matching. Before matching, the absolute value of standardised difference were greater than 0.1 for some of the covariates. After matching the covariates, the absolute standardised difference for covariates were less than 0.1 , indicating a balance in differences between the appropriate feeding groups.

\section{Appropriate complementary feeding estimation}

Table 4 presents the estimated odds ratios (ORs) with associated $95 \%$ confidence interval (CI) for the association of appropriate complementary feeding and wasting, underweight or stunting, using the unmatched and matched sample. For the unmatched sample, children who were appropriately complementary fed were less likely to be stunted $(\mathrm{OR}=0.6,(95 \% \mathrm{CI}: 0.4,0.9))$ and underweight $(\mathrm{OR}=0.5,(95 \%$ CI:0.3,0.96)). Using the matched sample, children who were on appropriate complementary feeding were less likely $(\mathrm{OR}=0.5,(95 \% \mathrm{CI}: 0.3,0.9))$ to be stunted compared to those who were not on appropriate complementary feeding. However, appropriate 
Table 3 Distribution of characteristics between appropriately and inappropriately fed children before and after matching

\begin{tabular}{|c|c|c|c|c|c|c|}
\hline \multirow[b]{3}{*}{ Characteristic } & \multicolumn{3}{|c|}{ Before Matching } & \multicolumn{3}{|c|}{ After Matching } \\
\hline & \multicolumn{3}{|c|}{ Appropriate $(n=788)$ Not Appropriate $(n=3934)$} & \multicolumn{3}{|c|}{ Appropriate $(n=788)$ Not appropriate $(n=788)$} \\
\hline & $\mathrm{n}(\%)$ & $\mathrm{n}(\%)$ & $p$ & $\mathrm{n}(\%)$ & $\mathrm{n}(\%)$ & $p$ \\
\hline \multicolumn{7}{|l|}{ Maternal HIV } \\
\hline HIV-uninfected & $620(78.7)$ & $3191(81.1)$ & & $620(78.7)$ & $610(77.4)$ & \\
\hline HIV-infected & $168(21.3)$ & $743(18.9)$ & 0.037 & $168(21.3)$ & $178(22.6)$ & 0.543 \\
\hline \multicolumn{7}{|l|}{ Age of child } \\
\hline 6-11 months & $229(29.1)$ & $1224(31.1)$ & & $229(29.1)$ & $237(30.1)$ & \\
\hline 12-17 months & $253(32.1)$ & $1283(32.6)$ & & $253(32.1)$ & $268(34.0)$ & \\
\hline $18-23$ months & $306(38.8)$ & $1427(36.3)$ & 0.346 & $306(38.8)$ & 283(35.9) & 0.480 \\
\hline \multicolumn{7}{|l|}{ Sex of child } \\
\hline Male & $368(45.6)$ & $2047(52.0)$ & & $376(50.3)$ & $379(48.1)$ & \\
\hline Female & $439(54.4)$ & $1887(48.0)$ & 0.006 & $392(49.7)$ & $409(51.9)$ & 0.392 \\
\hline \multicolumn{7}{|l|}{ Residence } \\
\hline Urban & $135(16.8)$ & $447(11.4)$ & & $139(17.6)$ & $146(18.5)$ & \\
\hline Rural & $672(83.2)$ & $3487(88.6)$ & $<0.001$ & $649(82.1)$ & $642(81.5)$ & 0.679 \\
\hline \multicolumn{7}{|l|}{ Mothers' Education } \\
\hline None & $105(13.3)$ & $619(15.7)$ & & 105(13.3) & $109(13.8)$ & \\
\hline Primary & $494(62.7)$ & $2581(65.6)$ & & $494(62.7)$ & $508(64.5)$ & \\
\hline Secondary \& Post-Secondary & $189(23.9)$ & $734(18.7)$ & 0.002 & $189(23.9)$ & $171(21.7)$ & 0.557 \\
\hline \multicolumn{7}{|l|}{ History of diarrhoea } \\
\hline Yes & $298(36.9)$ & $1350(34.0)$ & & $519(65.9)$ & $531(67.4)$ & \\
\hline No & $508(62.9)$ & $2579(65.6)$ & & $268(34.0)$ & $256(32.5)$ & \\
\hline Don't know & $1(0.1)$ & $5.0(0.1)$ & 0.951 & $1(0.1)$ & $1(0.1)$ & 0.814 \\
\hline \multicolumn{7}{|l|}{ Birth weight } \\
\hline Normal weight & $710(90.1)$ & $3548(90.2)$ & & $710(90.1)$ & $701(88.9)$ & \\
\hline Low birth weight & $78(9.9)$ & $386(9.8)$ & 0.05 & $78(9.9)$ & $87(11.1)$ & 0.459 \\
\hline \multicolumn{7}{|l|}{ Wealth } \\
\hline Poor & $329(40.8)$ & $1832(46.6)$ & & $305(39.6)$ & $266(40.5)$ & \\
\hline Medium & $117(14.5)$ & 705(17.9) & & $116(17.4)$ & $160(16.8)$ & \\
\hline Rich & $360(44.6)$ & $1397(35.5)$ & $<0.001$ & $367(42.9)$ & $362(42.7)$ & 0.932 \\
\hline \multicolumn{7}{|l|}{ Maternal Age } \\
\hline $15-24$ years & $323(40.0)$ & $1618(41.1)$ & & $323(39.6)$ & $366(44.0)$ & \\
\hline $25-34$ years & $336(41.6)$ & $1390(35.3)$ & & $336(41.6)$ & $289(34.7)$ & \\
\hline $35-49$ years & $148(18.4)$ & $926(23.5)$ & 0.005 & $148(18.4)$ & $177(21.3)$ & 0.116 \\
\hline
\end{tabular}

complementary feeding had no significant effect on wasting and underweight for the matched sample.

Table 4 Effect of complementary feeding on child growth indicators for children aged 6-23 months

\begin{tabular}{|c|c|c|c|c|}
\hline & & \multicolumn{3}{|c|}{ 2015-16 MDHS } \\
\hline & & Wasting & Stunting & Underweight \\
\hline & & OR $(95 \% \mathrm{Cl})$ & OR $(95 \% \mathrm{Cl})$ & OR(95\% Cl) \\
\hline & Not appropriate & 1.00 & 1.00 & 1.00 \\
\hline Unmatched & Appropriate & $1.1(0.7,2.4)$ & $0.6(0.4,0.9)$ & $0.5(0.3,0.96)$ \\
\hline Matched & Appropriate & $1.5(0.6,3.9)$ & $0.5(0.3,0.9)$ & $0.7(0.4,1.1)$ \\
\hline
\end{tabular}

1.00 - reference category 


\section{Sensitivity analysis}

Table 6 in the appendix presents the results of the Mantel-Haenszel test for stunting, wasting and underweight. For stunting, we observe that the critical value of underestimating stunting among the appropriately complementary fed and inappropriately complementary fed children was significant at an odds ratio of $1(p=0.025)$ and then at an odds ratio of $2.6(p=0.09)$. We note that the exposure effect on stunting is slightly unstable. This may suggest that the results on stunting are prone to underestimation by unobserved confounders. However, for the outcome wasting, the critical value of overestimating the exposure effect was significant at an odds ratio of $3.0(p=0.08)$. As for underweight, the effect of appropriate complementary feeding would be significant and sensitive to unobserved confounders at an odds ratio of 2.0. This implies that the results on wasting and underweight were robust against unobserved confounders as compared to the results for stunting.

\section{Discussion}

Appropriate complementary feeding is essential for a child's growth and development. This study set out to assess the causal association between appropriate complementary feeding and child growth in Malawi. Three indicators of growth namely: stunting, wasting and underweight were analysed. To our knowledge, this is the first study that assessed the effect of appropriate complementary feeding using a nationally representative survey and applying robust statistical methods such as the propensity score matching to balance confounders.

The propensity score matching method was able to balance confounders between children who were appropriately complementary fed and those who were not appropriately complementary fed. Appropriate complementary feeding had a positive effect on stunting and had no benefit on wasting and underweight. Our findings are similar to what has been found from previous studies that provision of complementary feeding significantly improved stunting and underweight among children less than 2 years $[15,47]$. In contrast, a study done in Bangladesh on infant and young child feeding practice among children aged 0-23 months found that children fed with adequate dietary diverse foods were negatively associated with stunting [16]. One of the possible reasons of having improved stunting may be because as observed from the study, a majority of the children were still breastfeeding and 
most of them were fed grains followed by legumes and nuts and they were fed more than two to three times. Since these foods are known to be a good source of fibre, carbohydrates and proteins $[48,49]$, combined with adequate feeding this may have helped in improving stunting in children aged 6 to 23 months. Previous studies have found that the incidence of stunting is high in children aged 6-24 months, since this is when a child has high demand for nutrients, at the same time limitations in the quantity and quality of available food exists especially among children from LMICs [47, 50]. However, our study provides evidence that ascertains the benefits of practising appropriate complementary feeding to reduce stunting in Malawi.

Our study found no association between appropriate complementary feeding and wasting and underweight. This is consistent with findings from a study done in Nepal where no association was found between complementary feeding practices and child nutritional status [51]. However, recent findings from a study done using the 2015-16 MDHS data found that children aged 0-23 months who met minimum meal frequency and minimum acceptable diet were less likely to be underweight [19]. We observe that the prevalence of children who received a minimum acceptable diet was low for our study and most of the children were from the rural areas where poverty is high and poor sanitation is rampant. Adherence to appropriate complementary feeding in resource limited settings like Malawi combined with high HIV burden is known to be a challenge [19, 37]. Previous studies have found that poverty, food insecurity due to lack of enough resources, unsafe water, poor sanitation and hygiene are associated with a high percentage of under-nutrition [11, 21, 52]. Guidelines implemented by the Ministry of Health $(\mathrm{MoH})$ of Malawi on Infant and young child feeding (IYCF) emphasise the need to follow proper hygiene when preparing food for a child $[10,53]$, however, a follow-up on whether mothers use proper hygiene and sanitation is not done in the country. Therefore, improper handling and preparation of the food may have resulted in diarrhoea and illnesses in the child which may have contributed to appropriate complementary feeding having no benefit on wasting and underweight.

Access and utilisation of postnatal and PMTCT services in Malawi has been found to be low $[12,19]$, even though $\mathrm{MoH}$ guidelines emphasize the need to increase access to nutrition services for mothers, especially among those who are HIV-infected [10]. 
Constant counselling and information sharing is important for mothers, especially among those who are HIV-infected. Using counselling cards community nutrition workers educate mothers on the concepts and approach of appropriate infant and young child feeding for effective practice [6]. However, mothers may not understand the information given to them during the clinic sessions [12]. In addition, a mothers' decision on when to initiate complementary feeding is influenced by the immediate families especially if a mother is HIV-infected [12], hence making it difficult for a mothers to follow recommended guidelines.

The major strength of this study is that we used the propensity score (PS) matching to account for confounding and control for selection bias. Comparability of exposure groups in terms of their measured covariates in this study was achieved. In addition, data originating from a large survey was used and hence, using a rich data with a vast number of socio-demographic characteristics of participants. Nonetheless, the study had limitations. Analysis was done on cross-sectional data and despite controlling for confounding, it was difficult to assess the causal relationship. The assumption of no unobserved confounding cannot be formally tested [42] thus, selection bias might still be present. In addition, knowledge on date of HIV acquisition and HIV test results was not known hence it may be possible that a mother was infected with HIV after a child was born. In this case, a mothers' HIV status would not be directly linked to a child's nutritional status indicator.

\section{Conclusion}

The study has shown that appropriate complementary feeding improves child growth, particularly stunting, among children aged 6-23 months. Statistical methods such as propensity score matching that balance confounder variables could be a useful tool to reduce bias in the estimation of exposure effects on health outcomes in observational studies. Our findings render more evidence on the policies and guidelines suggested by the $\mathrm{WHO}$ and $\mathrm{MoH}$, on the importance of following an appropriate complementary feeding practice for optimal child growth and development. We recommend promoting child nutrition education among mothers and care-takers to facilitate optimal feeding for infants and young children especially in a high HIV and resource limited settings. 


\section{List of abbreviations}

BF - Breastfeeding CF - Complementary feeding

HIV - Human Immune-deficiency Virus

IYCF - Infant and young child feeding

LMIC - Low-middle income countries

MDHS - Malawi Demographic and Health Survey

$\mathrm{MoH}$ - Ministry of Health

NN - Nearest Neighbour

PMTCT - Prevention of Mother to Child Transmission

PS - Propensity Score

PSM - Propensity Score Matching

SSA - Sub-saharan Africa

WHO - World Health Organisation

\section{Declaration}

Ethics approval and consent to participate

The Malawi Health Research Committee determined that ethical approval was not deemed necessary in this study considering the fact that the study used data from a research study already approved by an ethical research committee. According to the 2015-16 MDHS report [36], the MDHS study was ethically approved by Malawi Health Research Committee, Institutional Review Board of ICF Macro, Centre for Disease and Control (CDC) in Atlanta,

GA, USA and Prevention IRB. Informed consent was obtained from all eligible persons

Consent for Publication

Not applicable

Availability of data and materials

The data that support the findings of this study are available upon request from the Demographic and Health

Survey (DHS) website. Upon approval, full access is granted to all unrestricted survey datasets.

Competing interests

The authors declare that they have no competing interests.

\section{Funding}

This work was supported through the DELTAS Africa Initiative, Sub-Saharan Africa Consortium for Advanced Biostatistics Training (SSACABT). The DELTAS Africa Initiative is an independent funding scheme of the African Academy of Sciences (AAS)'s Alliance for Accelerating Excellence in Science in Africa (AESA) and supported by the New Partnership for Africa's Development Planning and Coordinating Agency (NEPAD Agency) with funding from the Wellcome Trust [SSACABT] and the UK government. Special thanks to South Africa Medical Research Council for hosting and supporting my research visits. The views expressed in this publication are those of the author(s) and not those of AAS, NEPAD Agency, Wellcome Trust or the UK government.

Author's contributions

HT performed data management, statistical analysis, and wrote the initial draft of manuscript. SM conceived and suggested the direction for this paper, reviewed statistical analysis and results and helped with the revision of the manuscript. DM helped with the concept and direction. All authors have read and approved the final manuscript. 


\section{Acknowledgements}

We acknowledge the DHS team for making the MDHS data available for use.

\section{Author details}

${ }^{1}$ Department of Mathematical Sciences, University of Malawi, Zomba, Malawi. ${ }^{2}$ Biostatistics Research Unit, South

Africa Medical Research council, Pretoria, South Africa. ${ }^{3}$ School of Mathematics, Statistics and Computer Science, University of Kwazulu-Natal, Pietermaritzburg, South Africa. ${ }^{4}$ Department of Statistics, University of Pretoria,

Pretoria, South Africa. ${ }^{5}$ Department of Statistics, University of Pennyslvania, Pennyslvania, USA.

\section{References}

1. UNICEF, et al.: Levels and trends in child malnutrition unicef-who-world bank group joint child malnutrition estimates: key findings of the 2015 edition. New York: UNICEF, WHO, World Bank Group (2015)

2. WHO, et al.: Global targets 2025 to improve maternal, infant and young children nutrition. Technical report, World Health Organization (2017)

3. UNICEF, et al.: Levels and trends in child malnutrition unicef-who-world bank group joint child malnutrition estimates: key findings of the 2015 edition. New York: UNICEF, WHO, World Bank Group (2016)

4. Duggan, M.: Anthropometry as a tool for measuring malnutrition: impact of the new who growth standards and reference. Annals of tropical paediatrics 30(1), 1-17 (2010)

5. De Onis, M., Blossner, M., Organization, W.H., et al.: Who global database on child growth and malnutrition. Technical report, World Health Organization (1997)

6. Dewey, K.: Guiding principles for complementary feeding of the breastfed child (2003)

7. WHO: Global Strategy for Infant and Young Child Feeding. World Health Organization, ??? (2003)

8. WHO, et al.: Indicators for assessing infant and young child feeding practices: part 2: measurement (2010)

9. WHO, et al.: Infant and Young Child Feeding: Model Chapter for Textbooks for Medical Students and Allied Health Professionals. Geneva: World Health Organization, ??? (2009)

10. Malawi Ministry of Health, P.: Infant and young child nutrition policy and guidelines $2003-2020$. Technical report, Malawi Ministry of Health and Population (2011)

11. Vaahtera, M., Kulmala, T., Hietanen, A., Ndekha, M., Cullinan, T., Salin, M.-L., Ashorn, P.: Breastfeeding and complementary feeding practices in rural malawi. Acta Paediatrica 90(3), 328-332 (2001)

12. Chintalapudi, N., Hamela, G., Mofolo, I., Maman, S., Hosseinipour, M.C., Hoffman, I.F., Flax, V.L.: Infant and young child feeding decision making and practices: Malawian mothers' and fathers' roles in the context of hiv. Journal of Human Lactation 34(1), 68-76 (2018)

13. Adetunji, J.: Trends in under-5 mortality rates and the hiv/aids epidemic. Bulletin of the World Health Organization 78, 1200-1206 (2000)

14. Perkins, J.M., Jayatissa, R., Subramanian, S.: Dietary diversity and anthropometric status and failure among infants and young children in sri lanka. Nutrition 55, 76-83 (2018)

15. Lassi, Z.S., Rind, F., Irfan, O., Hadi, R., Das, J.K., Bhutta, Z.A.: Impact of infant and young child feeding (iycf) nutrition interventions on breastfeeding practices, growth and mortality in low-and middle-income countries: systematic review. Nutrients 12(3), 722 (2020)

16. Zongrone, A., Winskell, K., Menon, P.: Infant and young child feeding practices and child undernutrition in bangladesh: insights from nationally representative data. Public health nutrition 15(9), 1697-1704 (2012)

17. Muhangi, L., Lule, S.A., Mpairwe, H., Ndibazza, J., Kizza, M., Nampijja, M., Nakazibwe, E., Kihembo, M., Elliott, A.M., Webb, E.L.: Maternal hiv infection and other factors associated with growth outcomes of hiv-uninfected infants in entebbe, uganda. Public health nutrition 16(9), 1548-1557 (2013)

18. Rossouw, M.E., Cornell, M., Cotton, M.F., Esser, M.M.: Feeding practices and nutritional status of hiv-exposed and hiv-unexposed infants in the western cape. Southern African journal of HIV medicine 17(1) (2016)

19. Walters, C.N., Rakotomanana, H., Komakech, J.J., Stoecker, B.J.: Maternal determinants of optimal breastfeeding and complementary feeding and their association with child undernutrition in malawi (2015-2016). BMC public health 19(1), 1503 (2019)

20. Manda, S.O., Kandala, N.-B., Ghilagaber, G.: Advanced techniques for modelling maternal and child health in africa. In: Advanced Techniques for Modelling Maternal and Child Health in Africa, pp. 1-7. Springer, ??? (2014) 
21. Kassa, T., Meshesha, B., Haji, Y., Ebrahim, J.: Appropriate complementary feeding practices and associated factors among mothers of children age 6-23 months in southern ethiopia, 2015. BMC pediatrics 16(1), 131 (2016)

22. Nkoka, O., Mhone, T.G., Ntenda, P.A.: Factors associated with complementary feeding practices among children aged 6-23 mo in malawi: an analysis of the demographic and health survey 2015-2016. International health 10(6), 466-479 (2018)

23. Dhami, M.V., Ogbo, F.A., Osuagwu, U.L., Agho, K.E.: Prevalence and factors associated with complementary feeding practices among children aged 6-23 months in india: a regional analysis. BMC public health $19(1)$, 1034 (2019)

24. Li, Z., Kim, R., Vollmer, S., Subramanian, S.: Factors associated with child stunting, wasting, and underweight in 35 low-and middle-income countries. JAMA Network Open 3(4), 203386-203386 (2020)

25. Rosenbaum, P.R., Rubin, D.B.: The central role of the propensity score in observational studies for causal effects. Biometrika 70(1), 41-55 (1983)

26. Lunceford, J.K., Davidian, M.: Stratification and weighting via the propensity score in estimation of causal treatment effects: a comparative study. Statistics in medicine 23(19), 2937-2960 (2004)

27. Rubin, D.B., Thomas, N.: Matching using estimated propensity scores: relating theory to practice. Biometrics, 249-264 (1996)

28. Cole, S.R., Hernan, M.A.: An Introduction to Propensity Score Methods for Reducing the Effect of Confounding in Observational. American Journal of Epidemiology (2008)

29. Austin, P.C.: An Introduction to Propensity Score Methods for Reducing the Effect of Confounding in Observational. Multivariate Behavioural Research (2011)

30. Rosenbaum, P.R., Rubin, D.B.: Constructing a control group using multivariate matched sampling methods that incorporate the propensity score. The American Statistician 39(1), 33-38 (1985)

31. He, H., Hu, J., He, J.: Overview of propensity score methods. In: Statistical Causal Inferences and Their Applications in Public Health Research, pp. 29-48. Springer, ??? (2016)

32. Sainani, K.L.: Propensity scores: uses and limitations. PM\&R 4(9), 693-697 (2012)

33. Austin, P.C., Mamdani, M.M.: A comparison of propensity score methods: a case-study estimating the effectiveness of post-ami statin use. Statistics in medicine 25(12), 2084-2106 (2006)

34. d'Agostino, R.B.: Propensity score methods for bias reduction in the comparison of a treatment to a non-randomized control group. Statistics in medicine 17(19), 2265-2281 (1998)

35. Bai, H.: A comparison of propensity score matching methods for reducing selection bias. International Journal of Research \& Method in Education 34(1), 81-107 (2011)

36. Government of Malawi, N., ICF: Malawi demographic and health survey 2015-16 report. Technical report, National Statistics Office Malawi and ICF (2017)

37. Chinkonde, J.R., Hem, M.H., Sundby, J.: Hiv and infant feeding in malawi: public health simplicity in complex social and cultural contexts. BMC Public Health 12(1), 1-9 (2012)

38. Organization, W.H., et al.: WHO Child Growth Standards: Length/height-for-age, Weight-for-age, Weight-for-length, Weight-for-height and Body Mass Index-for-age: Methods and Development. World Health Organization, ??? (2006)

39. Kamenju, P., Liu, E., Hertzmark, E., Spiegelman, D., Kisenge, R., Kupka, R., Aboud, S., Manji, K.P., Duggan, C., Fawzi, W.W.: Nutritional status and complementary feeding among hiv-exposed infants: a prospective cohort study. Maternal \& child nutrition 13(3), 12358 (2017)

40. Twabi, H.S., Manda, S.O., Small, D.S.: Assessing the effects of maternal hiv infection on pregnancy outcomes using cross-sectional data in malawi. BMC Public Health (2020)

41. Kurth, T., Walker, A.M., Glynn, R.J., Chan, K.A., Gaziano, J.M., Berger, K., Robins, J.M.: Results of multivariable logistic regression, propensity matching, propensity adjustment, and propensity-based weighting under conditions of nonuniform effect. American journal of epidemiology 163(3), 262-270 (2006)

42. Hernán, M.A., Robins, J.M.: Causal inference. What If. CRC Boca Raton, FL; (2020)

43. Austin, P.C.: Balance diagnostics for comparing the distribution of baseline covariates between treatment groups in propensity-score matched samples. Statistics in medicine 28(25), 3083-3107 (2009)

44. Normand, S.-L.T., Landrum, M.B., Guadagnoli, E., Ayanian, J.Z., Ryan, T.J., Cleary, P.D., McNeil, B.J.: 
Validating recommendations for coronary angiography following acute myocardial infarction in the elderly: a matched analysis using propensity scores. Journal of clinical epidemiology 54(4), 387-398 (2001)

45. Aakvik, A.: Bounding a matching estimator: the case of a norwegian training program. Oxford bulletin of economics and statistics 63(1), 115-143 (2001)

46. Rosenbaum, P.R.: Sensitivity analysis in observational studies. Statistics Reference Online 4, 1809-1814 (2005)

47. Lassi, Z.S., Das, J.K., Zahid, G., Imdad, A., Bhutta, Z.A.: Impact of education and provision of complementary feeding on growth and morbidity in children less than 2 years of age in developing countries: a systematic review. BMC public health 13(S3), 13 (2013)

48. Messina, M.J.: Legumes and soybeans: overview of their nutritional profiles and health effects. The American journal of clinical nutrition 70(3), 439-450 (1999)

49. Committee, M.V.A.: Market assessment report. Technical report (2014)

50. Dewey, K.G., Adu-Afarwuah, S.: Systematic review of the efficacy and effectiveness of complementary feeding interventions in developing countries. Maternal \& child nutrition 4, 24-85 (2008)

51. Pokharel, P., Adhikari, A., Lamsal, P., Adhikari, R.: Effect of complementary feeding practices and nutritional status of children (6-23 months) in tamang community, ambhanjyang vdc of makwanpur. Janaki Medical College Journal of Medical Science 5(1), 22-32 (2017)

52. Seetha, A., Tsusaka, T.W., Munthali, T.W., Musukwa, M., Mwangwela, A., Kalumikiza, Z., Manani, T., Kachulu, L., Kumwenda, N., Musoke, M., et al.: How immediate and significant is the outcome of training on diversified diets, hygiene and food safety? an effort to mitigate child undernutrition in rural malawi. Public Health Nutrition 21(6), 1156-1166 (2018)

53. Phiri, F.P.: Experiences of multi-sector programming in malawi. Field Exchange 52, 97 (2016)

54. Udoh, E.E., Amodu, O.K.: Complementary feeding practices among mothers and nutritional status of infants in akpabuyo area, cross river state nigeria. SpringerPlus 5(1), 2073 (2016)

55. Andare, N., Ochola, S., Chege, P.: Determinants of infant feeding practices among mothers living with hiv attending prevention of mother to child transmission clinic at kiambu level 4 hospital, kenya: a cross-sectional study. Nutrition Journal 18(1), 64 (2019)

Tables

Table 5 Absolute Standard Differences and p-values Before and After Application of Propensity Matching

\begin{tabular}{lcccc}
\hline & \multicolumn{2}{c}{ Before } & \multicolumn{2}{c}{ After } \\
\cline { 2 - 5 } Confounder & Abs. Std Diff & p-value & Abs. Std Diff & p-value \\
\hline Sex of a child & 0.03 & 0.77 & 0.08 & 0.957 \\
Mothers' education & 0.16 & 0.629 & 0.05 & 0.513 \\
childs' age & 1.22 & 0.003 & 0.003 & 0.426 \\
source of drinking water & 0.03 & 0.002 & 0.04 & 0.256 \\
Food type & 1.04 & 0.002 & 0 & 0.02 \\
Feeding counselling & 0.07 & 0.424 & 0.04 & 0.37 \\
residence & 0.06 & 0.11 & 0.04 & 0.825 \\
Had diarrhoea & 0.27 & 0.034 & 0.04 & 0.179 \\
Had Vitamin A & 0.18 & 0.508 & 0.02 & 0.642 \\
Region & 0.01 & 0.784 & 0.05 & 0.303 \\
Mothers' employment & 0.04 & 0.409 & 0.06 & 0.197 \\
Wealth & 0.05 & 0.186 & 0.01 & 0.592 \\
Mothers' age & 0.18 & 0.002 & 0.01 & 0.417 \\
Birth weight & 0.003 & 0.155 & 0.04 & 0.064 \\
Size at birth & 0.02 & 0.504 & 0.08 & 0.369 \\
Antenatal visit & 0.05 & 0.75 & 0 & 0.486 \\
\hline
\end{tabular}


Table 6 Sensitivity Analyses for Unobserved Confounding

\begin{tabular}{lccc}
\hline Gamma & $\begin{array}{c}\text { Q- } M H^{-} \\
\text {Underweight }\end{array}$ & $\begin{array}{c}\text { Wasting } \\
\text { Q- } M H^{+}\end{array}$ & $\begin{array}{c}\text { Stunting } \\
\text { Q- } M H^{-}\end{array}$ \\
\hline 1 & 0.53 & 0.38 & $1.95^{* *}$ \\
1.2 & -0.10 & 0.02 & 1.26 \\
1.4 & 0.33 & -0.20 & 0.68 \\
1.6 & 0.78 & 0.10 & 0.19 \\
1.8 & 1.18 & 0.33 & -0.02 \\
2 & $1.54^{*}$ & 0.55 & 0.37 \\
2.2 & $1.87^{* *}$ & 0.76 & 0.71 \\
2.4 & $2.18^{* *}$ & 0.95 & 1.03 \\
2.6 & $2.46^{* * *}$ & 1.12 & $1.33^{*}$ \\
2.8 & $2.7^{* * *}$ & $1.28^{*}$ & $1.61^{*}$ \\
3 & $2.9^{* * *}$ & $1.48^{*}$ & $1.86^{* *}$ \\
\hline
\end{tabular}

Data imputed using multiple imputation

Q- $M H^{+}$or Q- $M H^{-}$Mantel-Haenszel Statistic for overestimation and underestimation of the ATE.

$* p<0.10 ; * * p<0.05 ; * * * p<0.01$ 
Figures

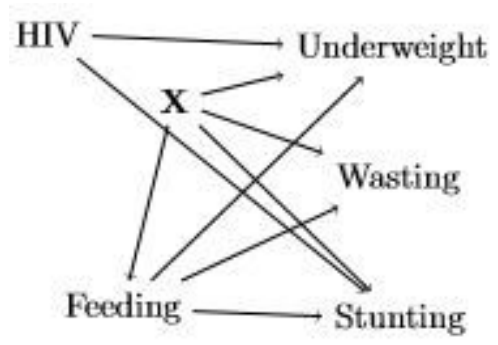

\section{Figure 1}

Causal pathway for Appropriate Feeding and Child Nutrition 